\title{
The Neural Correlates of Updating Information in Verbal Working Memory
}

\author{
Martial Van der Linden and Fabienne Collette \\ Neuropsychology Unit, University of Liège, Belgium \\ Eric Salmon \\ Cyclotron Research Centre, and Department of Neurology, University of Liège, Belgium \\ Guy Delfiore, Christian Degueldre, and A. Luxen \\ Cyclotron Research Centre, University of Liège, Belgium \\ G. Franck \\ Department of Neurology, University of Liège, Belgium
}

\begin{abstract}
The aim of the present study was to re-examine cerebral areas subserving the updating function of the central executive with a running span task requiring subjects to watch strings of consonants of unknown length and then to recall serially a specific number of recent items. In order to dissociate more precisely the updating process from the storage function, a four-item instead of a six-item memory load was used, contrary to our previous study (Salmon et al., 1996). In addition, a serial recall procedure was preferred to a recognition procedure in order to suppress the use of visuospatial strategies. The most significant increase of rCBF occurred in the left frontopolar cortex (Brodmann's area 10), spreading to the left middle frontal (Brodmann's area 46). Results suggest that frontopolar activation underlies an updating process in working memory.
\end{abstract}

\section{INTRODUCTION}

Working memory refers to a limited capacity system which is responsible for the processing and temporary storage of information. Baddeley's model represents a current and influential attempt to define the structure and functioning of working memory (Baddeley, 1986). This model comprises a modality-free controlling central executive which is aided by a number of peripheral slave systems ensuring temporary maintenance of information. Two such systems have been more deeply explored: the phonological loop and the visuospatial sketchpad. The visuospatial sketchpad system is assumed to be involved in setting up and maintaining visuospatial material. The phonological loop system provides temporary storage for speech-based material and is composed of two subsystems: a passive phonological input store and an active articulatory rehearsal process. The central executive is assumed to be an attentional control system responsible for strategy selection, planning, decision making, and for control and co-ordination of the various processes involved in short-term storage and more general processing tasks.

In recent years, a large number of neuroimaging studies have contributed to identifying the physiological substrate of the different components of working memory (for a review, see Smith \& Jonides, 1997). Several experiments have studied a variety of central executive processes such as random number generation (Petrides, Alivastos, Evans, \& Meyer, 1993a; Petrides, Alivastos, Meyer, \& Evans, 1993b) or co-ordination of dual tasks (D'Esposito et al., 1995). All these studies support the involvement of the dorsolateral prefrontal cortex (especially Brodmann's areas [BA] 46 and 9) in the central executive functioning.

However, a major difficulty in the exploration of the neural basis of the central executive is finding a task in which the role of the central executive can be clearly distinguished from that of the slave (storage) systems. In that perspective, the updating memory paradigm initially used by Pollack, Johnson, and Knaff (1959) and investigated by Morris and Jones (1990) appears to meet this requirement. The task requires subjects to watch strings of consonants of unknown length, and to recall serially a specific number of recent items. It requires considerable flexibility and a progressive shift of attention, i.e. discarding some items while new ones are registered. Morris and Jones (1990) showed that the updating memory task requires two independent mechanisms: the phonological loop and the central executive. The updating process requires central executive resources but not the phonological loop. Conversely, the serial recall (storage) component of the task requires the phonological loop but not the central executive.

In a recent PET study, we investigated the neural basis of this central executive updating process (Salmon et al., 1996) by using a working-memory updating task adapted from Morris and Jones (1990). In this task, lists of eight, nine, and ten consonants were presented at a rate of one per second. Subjects were not informed of the length of each list before presentation. They were asked to remember serially only the last six items (a six-item memory. load). In the phonological short-term memory (control) task, sequences of six consonants were presented and the subjects were instructed to remember them serially.

When the updating task (requiring both the central executive and the phonological loop) was compared with the phonological short-term memory task (requiring the phonological loop), activation in a set of frontal and parietal 
regions was observed. An increase of $\mathrm{rCBF}$ occurred in the right mid-dorsal prefrontal cortex (BA 9), in left middle frontal regions (BA 46 and possibly BA 10) and in the right frontal pole (BA 10). Increased rCBF was found in a broad area of the right inferior parietal arid angula gyri (BA 40/39), and in the left supramarginal gyrus (BA 40). We also observed activation foci in cuneus/ precuneus and superior occipital gyri on both sides (BA 18/19), in the right thalamus, and in the cerebellum.

However, a problem with this study was that we changed the Morris and Jones (1990) original updating procedure by converting the serial recall task into a short-term memory recognition task. This was made in order to be close to the procedure used by Paulesu, Frith, and Frackowiak (1993) to explore the neural substrates of the phonological loop. More specifically, instead of asking the subjects to recall serially the last six items of a sequence, they were asked to judge whether a consonant displayed two seconds after each list was present in the six last consonants for this particular list (a similar recognition procedure was used in the phonological shortterm memory task). We recently obtained preliminary data suggesting that the recognition and the recall procedures promote the preferential utilisation of visuospatial and phonological strategies, respectively. In addition, post hoc questioning of the subjects examined in our initial PET study indicated that half of them used a phonological strategy in the updating memory experiment, while the other half used a visual imagery strategy or a combination of phonological and visuospatial strategies. As it appears that the subjects have frequently adopted a visuospatial strategy in our experimental updating condition, some of the observed activation (e.g. parietal and occipital activation) might be related to generation and short-term storage of visuospatial images rather than to central executive functioning. Another problem was that the subjects were asked to remember the last six items, a memory load close to, or beyond their memory span. According to Baddeley (1986; see also Vallar \& Baddeley, 1984), span performance depends on both the phonological loop system and the central executive. The phonological loop system is able to store only a limited number of items but the central executive may increase this number either by improving the working of the phonological loop (for example, by grouping items into higher-level units) or even by using long-term memory information. In that perspective, it is plausible that holding a six-item memory load is very dependent on central executive resources and consequently, in our updating experiment, the central executive system would have been involved not only in the updating process but also in the storage function.

The present PET experiment was carried out to re-examine the brain regions involved in working-memory updating by using a serial recall procedure instead of a recognition procedure and by using a sub-span (fouritem) memory load which is presumably less dependent on the central executive.

\section{MATERIALS AND METHODS}

\subsection{Subjects}

Six male, European, right-handed volunteers (age range 20-25 years) gave written informed consent to take part in this study, which was approved by the University of Liège Ethics Committee. None had any past medical history nor used any medication.

\subsection{PET Scanning}

Scans of regional cerebral blood flow (rCBF) were obtained for each subject using a CTI model 951/31 R PET scanner (CTI, Knoxville, Tenn., USA) with collimating septa extended. The physical characteristics of the tomograph have been described previously (Degueldre \& Quaglia, 1992). Subjects wore an individual thermoplastic face mask for head stabilisation. A transmission scan was acquired for attenuation correction using three rotating sources of $68 \mathrm{Ge}$. Emission scans were reconstructed using a Hanning filter at a cut-off frequency of 0.5 cycles per pixels giving a transaxial resolution of $8.7 \mathrm{~mm}$ full width at half maximum and an axial resolution of $5 \mathrm{~mm}$ for each of 31 planes with a total field of view of $10.8 \mathrm{~cm}$ in this direction.

Volunteers received a 60 seconds intravenous infusion of $\mathrm{H}_{2}{ }^{15} \mathrm{O}$ (total activity $35 \mathrm{mCi}$ ) through a left forearm cannula. A dynamic PET scan consisting of two frames was collected over a period of three minutes (background frame duration, one minute, second frame duration, two minutes). The infusion of ${ }^{15} \mathrm{O}$ labelled water began 45 seconds after acquisition start time (Lammersta et al., 1990). Cognitive activation started upon $\mathrm{H}_{2}{ }^{15} \mathrm{O}$ infusion, 15 seconds before the second scan. The integrated counts per pixel recorded during the second scan were used as an index of rCBF (Fox \& Mintum, 1989; Mazziota et al., 1985). All subjects underwent six consecutive $\mathrm{rCBF}$ measurements (three for each experimental and control condition). Fifteen minutes elapsed between the scans. The order of memory tasks was fixed so that control and experimental tasks alternated.

\subsection{Memory Tasks}

In the control verbal working-memory task (see Fig. Ia), randomised sequences of four consonants were 
displayed on a computer screen at a rate of one per second. Subjects were instructed to rehearse the stimuli silently and to remember them serially, in order to repeat the sequence aloud after presentation of each list (the end of the list being indicated by a question mark). The presentation of each sequence began after the subjects pressed a key-response.

In the updating working-memory task (adapted from Morris \& Jones, 1990; see also Van der Linden, Brédart, \& Beerten, 1994; see Fig. Ib), lists of 4, 6, 8, 10 consonants were presented at a rate of one per second. Subjects were not informed of the length of each list before presentation. They were asked to rehearse silently and to remember serially only the last four items. They had to repeat those four items aloud after presentation of each list (the end of the list being indicated by a question mark). The presentation of each sequence began after the subjects pressed a key-response.

Sequences sounding like words and abbreviations were avoided, and for the updating task, the various lists were presented in a randomised order, with the restriction that no more than two lists of the same length were presented successively. Subject responses were recorded on a tape microphone. Patients were trained five or six days before the PET session. Five minutes before each acquisition, instructions were rehearsed. The control task consisted of 16 sequences, the experimental task of 10 sequences, each task lasting between 210 and 240 seconds.

\subsection{Data Analysis}

Image analysis was performed on a SPARC workstation (Sun Microsystems Inc., Surrey, UK) using statistical parametric mapping software 95 (Friston et al., 1995). Each reconstructed rCBF scan consisting of 31 primary transverse planes was interpolated to 43 planes to render the voxels isotropic. The six acquisitions from each subject were realigned using the first as reference (Woods, Cherry, \& Mazziotta, 1992). The data were then transformed into a standard stereotactic space (Talairach \& Tournoux, 1988). A gaussian filter (16mm full width at half maximum) was applied to smooth each image to accommodate inter-subject differences in gyral and functional anatomy and to suppress high-frequency noise in the images. Such transformation of the data enables pixel-by-pixel averaging of data across subjects and for direct cross-reference to the anatomical features in the standard stereotactic atlas.

Differences in global activity within and between subjects were removed by analysis of covariance on a pixelby-pixel basis with global count as covariate and regional activity across subjects for each task as treatment (Friston et al., 1990). The across-task comparisons were first performed by averaging paired measurements. For each pixel in stereotactic space, the analysis of covariance (ANCOVA) generated a condition-specific adjusted mean $\mathrm{rCBF}$ value (normalised to $50 \mathrm{ml} / 100 \mathrm{ml} / \mathrm{min}$ ) and an associated adjusted error variance. The ANCOVA enabled comparison of the means across conditions on a pixel-by-pixel basis using the $t$ statistic. The resulting sets of $t$ values constituted statistical parametric maps [SPM(t)] (Friston, Frith, Liddle, \& Frackowiak, 1991). The SPM(t) were transformed to the unit normal distribution $[\operatorname{SPM}(Z)]$. The design of our study was the comparison of a task implicating both the phonological loop and the central executive to a task implicating only the phonological loop. Besides this main effect, we looked for time or position effects in the repeated measurements, and for interactions between task and "time" effects. For all analyses, we used a SPM thresholded at $P<.001$, with a further correction for multiple comparisons $(P<.05)$. We anticipated bilateral frontopolar activation and/or dorsolateral frontal activation in the updating task (Salmon et al., 1996). For this additional hypothesis-driven analysis, we used a statistical threshold of $\mathrm{P}<.05$, corrected for the number of regions interrogated $(n=4)$ (Poline, Holmes, Worsley, \& Friston, 1997).

(A)

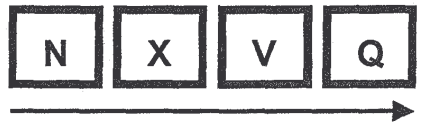

silent rehearsal

\section{(serial recall)}

(B)

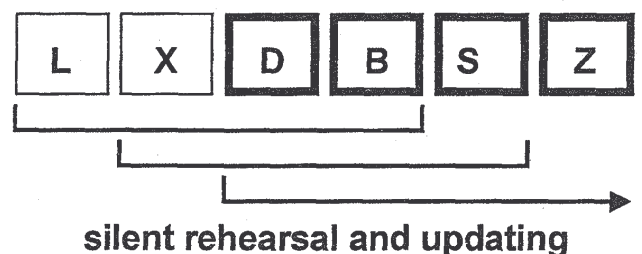

(serial recall)

FIG. 1. Schematic illustration of cognitive components involved in control and experimental tasks. 


\section{RESULTS}

\subsection{Neuropsychological Performance}

Subjects made only a few errors in the various tasks. There was no significant difference in the number of errors between the phonological short-term memory task (correctly recalled letters: $98.11+1.17 \%$ ) and the updating working-memory task (correctly recalled letters: $91.33 \pm 7.80 \% ; \mathrm{z}=1.75, P=.08$ ). Questioning the subjects about the way they carried out the tasks revealed that all subjects but one used verbal rehearsal, the last subject using mixed verbal and visual strategy. All subjects performed the updating task by removing successively the first letter(s) of the sequence for each presentation of a novel item after the fourth consonant was presented.

\subsection{PET Data}

When the updating working-memory task was compared with the phonological working-memory task, significant activation appeared in the left frontopolar cortex (BA 10), spreading to the left middle frontal cortex (BA 46). A significant activation was also observed in the right frontopolar cortex. Activation was located in the left frontopolar region when we used a SPM thresholded at $\mathrm{p}<.001$ (see Plate 1, and Table 1). In order to assess an eventual effect of task difficulty (as the difference between the error rates in the updating and control condition approached significance), the metabolic changes between these conditions were assessed with the error score as a confounding covariate. The cerebral areas found in that analysis were similar to those found in the first analysis $(\mathrm{P}<.01$, non-corrected for multiple comparisons).

A relative decrease of $\mathrm{rCBF}$ was observed in the right postcentral gyrus $(40,-20,32, \mathrm{Z}=4.58)$ for the updating working-memory task. There was also a trend for deactivation in the posterior cingulate cortex and the precuneus.

When looking for a temporal or position effect (Tables 2 and 3), we observed a decrease of rCBF from the initial to the last pair of tasks (first performance on both experimental and control tasks to the third performance on the tasks) in the left (fusiform) temporal inferior gyrus (BA 20). There was a rCBF increase (from the first to the third performance on the tasks) in the right postcentral (BA 1/3) and precentral (BA 4/6) gyri. The main point was that no significant interaction existed between task and time.

TABLE 1 Regions in Which There Was More Activation During Updating Condition Than Control Condition

\begin{tabular}{lllll} 
Area & $X$ & $Y$ & $Z$ & $Z$ score \\
\hline Left frontopolar (BA 10)* & -24 & 50 & 4 & 4.32 \\
Left middle frontal (BA 46)** & -50 & 24 & 24 & 3.15 \\
Right frontopolar (BA 10)*** & 30 & 56 & -4 & 3.57
\end{tabular}

Coordinates (in mm, relative to the anterior commissure) and $\mathrm{Z}$ scores. ${ }^{*} P$ value $<.001$, corrected for multiple comparisons. $* * P$ value $<.05$, non-corrected for multiple comparisons.

TABLE 2 Regions in Which There Was a Decreased Activation From First Performance on Both Experimental and Control Tasks to Last

\begin{tabular}{lllll} 
Area & $X$ & $Y$ & $Z$ & $Z$ score \\
\hline Left fusiform gyrus/inferior & -54 & -16 & 24 & 4.40
\end{tabular}

temporal (BA 20)

Coordinates (in mm, relative to the anterior commissure) and $\mathrm{Z}$ scores. $P$ value $<.001$, corrected for multiple comparisons.

TABLE 3 Regions in Which There Was an Increased Activation From First Performance on Both Experimental and Control Tasks to Last

\begin{tabular}{lcccc}
\hline Area & $X$ & $Y$ & $Z$ & $Z$ score \\
\hline Right post central gyrus (BA 1/3) & 46 & -18 & 44 & 5.12 \\
Right precential gyrus (BA 4) & 42 & -18 & 36 & 4.98 \\
Right precentral gyrus (BA 6) & 46 & -8 & 28 & 4,68
\end{tabular}




\section{DISCUSSION}

The aim of the present study was to re-examine the cerebral susbstrate of what appears to be an important function of the central executive component of working memory, i.e. the updating of information. In order to obtain a better dissociation between the updating process, requiring mainly central executive resources, and the storage function, requiring mainly the phonological loop, we used a serial recall procedure and a four-item memory load instead of a recognition procedure and a six-item memory load used in our previous study (Salmon et al., 1996).

The comparison of phonological short-term memory with working-memory updating confirmed that the prefrontal cortex is a key structure for central executive functioning. However, the most significant increase of rCBF occurred more specifically in the left frontopolar cortex (BA 10). Activation spread to the left middle frontal cortex (BA 46), and it was also observed in the right frontopolar cortex.

The dorsolateral prefrontal cortex (especially BA 9 and 46) is the region the most often implicated in workingmemory tasks (Cohen et al., 1994; Mellers et al., 1995; Petrides et al., 1993a, b). Several functions have been attributed to this region, such as the co-ordination of dual tasks (D'Esposito et al., 1995), the temporal coding of items (Cohen et al., 1997), storage functions (Braver et al., 1997; Smith, Jonides, \& Koeppe, 1996) or, in a more general way, the manipulation of information in working memory (Cohen et al., 1994 ; Owen, Evans, \& Petrides, 1996 ; Petrides et al., 1993a, b; Salmon et al., 1996). However, some of the working-memory tasks used in those studies were multi-compound and did not permit researchers to isolate a specific function of the central executive or to separate precisely executive function from storage processing. In the present study, a specific function of the central executive, the updating of information, was explored and the region the most implicated in that function was the left frontopolar cortex (BA 10).

Increase of $\mathrm{rCBF}$ in the frontopolar cortex has been reported in several other working-memory activation studies, along with a dorsolateral prefrontal cortex activation (Owen et al., 1996; Petrides et al., 1993b; Salmon et al., 1996), but the role of those regions in working-memory tasks has not been specifically discussed. An intervention of the frontopolar cortex was also found in word recall tasks when the number of items to memorise was near to or above the span level (Becker et al., 1994; Grasby et al., 1993, 1994). In this context, it could be argued that the frontopolar cortex is related to the contribution of the central executive in storage function when the subjects are submitted to a memory load close to or beyond the memory span (see Salmon et al., 1996). However, in the present study, it seems unlikely that the frontopolar cortex was implicated in a specific storage function. Indeed, the memory load used in the present study (four items) was low and would plausibly not require an intervention of the "storage" function of the central executive. In addition, Braver et al. (1997) and Smith and Jonides (1997) showed a linear increase of metabolic activity, related to the memory load, in the prefrontal dorsolateral cortex bilaterally (BA 9/46), and not in the frontopolar cortex. Finally, it should be noted that no frontopolar cortex activation has been related to the phonological loop of working memory. Indeed, previous studies (e.g. Salmon et al., 1996; Paulesu et al., 1993) showed that the neural substrates of tasks involving only the phonological loop were located in the premotor cortex and adjacent Broca's area (BA 6/44), in the left temporal gyrus (BA 22/42), in the left and right insula, and in the inferior part of the left inferior parietal gyrus (BA 40).

Accordingly, the frontopolar cortex activation observed in the present study could be specifically related to the updating of working memory. The function of this updating process is to continuously modify the content of working memory according to newer external (sensory input) or internal (long-term memory retrieval) information. Interestingly, Grasby et al. (1994) showed that activation in the frontopolar cortex increased in relation to the number of times that subjects had to repeat a single word. It appears that this task also involves a continuous updating process, that is to control how many times the word was repeated and had again to be repeated, and the updating process becomes more important as the number of repetitions increases. However, it remains to be determined whether the activation observed in the frontopolar cortex is related to a general updating process (independent of the type of updated information; i.e. semantic, visual, or phonological) or to an updating operation specifically devoted to serial verbal recall. Furthermore, it could be argued that the attribution of an updating function to the frontopolar cortex is not related to the specific involvement of the central executive component but rather to a greater difficulty in the updating task than in the serial recall task. Accordingly, recall performance tended to be lower in the updating task than in the serial recall task. However, when the error score of the subjects is taken as the covariate, similar activations were observed in the comparison of the updating and serial recall task.

In conclusion, the present study shows that, when the influence of the storage function is removed, specific cerebral regions can be isolated in tasks implicating the central executive, a structure nevertheless considered to have highly integrated functioning. In the same vein, Owen et al. (1996) demonstrated the existence of two functionally distinct subdivisions of the lateral frontal cortex, subserving different aspects of spatial working memory, with the ventrolateral frontal cortex (BA 47) related to the organisation and execution of a sequence of 
spatial moves and the mid-dorsolateral frontal cortex (BA 46/9) related to active monitoring and manipulation of spatial information. These findings suggest that the exploration of the central executive by means of functional imagery will be fruitful only if the experimental designs succeed in isolating specific components of this system. Moreover, given the integrative functioning of the central executive, the study of the relationships existing between several cerebral areas implicated in working-memory tasks will be particularly interesting. Indeed, it seems probable that the central executive function involves different interactions between a network of regions rather than specific regions devoted to each task. So, the frontopolar region appears to be involved in updating processes, but dorsolateral cortices are recruited when memory load increases. Moreover, the relative lateralisation of activation depends on the preferential use of verbal or visual strategies.

\section{REFERENCES}

Baddeley, A.D. (1986). Working memory. Oxford: Clarendon Press

Becker, J.T., Mintum, MA., Diehl, DI, Dobkin, J., Martidis, A., Madoff, D.C., \& DeKosky, S.T. (1994). Functional neuroanatomy of verbal free recall: A replication study. Human Brain Mapping, 1, 284-292.

Braver, T.S., Cohen, ID., Nystrom, L.E., Jonides, J., Smith, E.E., \& Noll, D.C. (1997). A parametric study of prefrontal cortex involvement in human working memory. Neuroimage, 5 , 49-62.

Cohen, J.D., Forman, S.D., Braver, T.S., Casey, B.J., Servan-Schreiber, D., \& Noll, D.C. (1994). Activation of the prefrontal cortex in a nonspatial working memory task with functional MRI. Human Brain Mapping, 1, 293-304.

Cohen, ID., Perlstein, W.M., Braver, T.S., Nystrom, L.E., Noll, D.C, Jonides, J., \& Smith, E.E. (1997). Temporal dynamics of brain activation during a working memory task. Nature, $386,604-607$.

Degueldre, C, \& Quaglia, L. (1992). Performance evaluation of a new whole body position tomograph: the ECAT 951/31 R. Proceedings of the 14th Annual International Conference of the IEEE. Engineering in Medicine and Biology Society, 14, 1831-1833.

D'Esposito, M., Detre, J.A., Alsop, CD., Shin, R.K., Atlas, S., \& Grossman, M. (1995). The neural basis of the central executive of working memory. Nature, 378, 279-281.

Fox, P.T, \& Mintum, M.A. (1989). Noninvasive functional brain mapping by change-distribution analysis of averaged PET images of $\mathrm{H}_{2}{ }^{15} \mathrm{O}$ tissue activity. Journal of Nuclear Medicine, 30, 141- 149.

Friston, KJ., Frith, CD., Liddle, P.F., Dolan, R.J., Lammeitsma, A.A.., \& Frackowiak, R.S.J. (1990). The relationship between global and local changes in PET scans. Journal of Cerebral Blood Flow and Metabolism, 10, 458-466.

Friston, KJ., Frith, CD., Liddle, P.F., \& Frackowiak, R.SJ. (1991). Comparing functional (PET) images: The assessment of significant changes. Journal of Cerebral Blood Flow and Metabolism, 11, 690-699.

Friston, KJ., Holmes, A.P., Worsley, K.J., Poline, J.-B.,, Frith, CD., \& Frackowiak, R.S.J. (1995). Statistical parametric maps in functional imaging: A general linear approach. Human Brain Mapping, 2, 189-210.

Grasby, P.M., Frith, CD., Friston, KJ., Bench, C, Frackowiak, R.S.J., \& Dolan, J.R. (1993). Functional mapping of brain areas implicated in auditory-verbal memory function. Brain, 116, 1-20.

Grasby, P.M., Frith, CD., Friston, K.J., Simpson, J., Fletcher, P.C, Frackowiak, R.S.J., \& Dolan, J.R. (1994). A graded task approach to the functional mapping of brain areas implicated in auditory-verbal memory. Brain, 117, 1271-1282.

Lammertsma, A.A., Cunningham, V.J., Deiber, M.P., Heather, J.D., Bloomfield, P.M., \& Nutt, J. (1990). Combination of dynamic and integral methods for generating reproducible functional CBF images. Journal of Cerebral Blood Flow and Metabolism, 10, 675-686.

Mazziotta, J.C, Huang, S.C, Phelps, M.E., Carson, RE., MacDonald, N.S., \& Mahoney, K. (1985). A noninvasive positron computed tomography technique using oxygen-15 labeled water for the evaluation of neurobehavioral task batteries. Journal of Cerebral Blood Flow and Metabolism, 5, 70-78.

Mellers, J.C.D., Bullmore, E., Brammei, M., Williams, S.C.R., Andrew, C, Sachs, N., Andrews, C, Cox, T.S., Simmons, A., Woodruff, P., David, A.S., \& Howard, R. (1995). Neural correlates of working memory in a visual letter monitoring task: An fMRI study. Neuroreport, 7 , 109-112.

Morris, N., \& Jones, D.M. (1990). Memory updating in working memory: The role of the central executive. British Journal of Psychology, 81, 111-121.

Owen, A.M., Evans, A.C., \& Petrides, M. (1996). Evidence for a two-stage model of spatial working memory processing within the lateral frontal cortex: A positon emission tomography study. Cerebral Cortex, 6, 31-38.

Paulesu, E., Frith, CD., \& Frackowiak, R.SJ. (1993). The neural correlates of the verbal component of working memory. Nature, 362, 342-345. 
Published in: Memory (1999), vol. 7, iss. 5-6, pp. 549-560

Status: Postprint (Author's version)

Petrides, M., Alivastos, B., Evans, A.C., \& Meyer, E. (1993a). Dissociation of human mid-dorsolateral from posterior dorsolateral frontal cortex in memory processing. Proceedings of National Academy of Sciences, USA, 90, 873-877.

Petrides, M., Alivastos, B., Meyer, E., \& Evans, A.C (1993b). Functional activation of the human frontal cortex during the performance of verbal working memory tasks. Proceedings of National Academy of Sciences, USA, 90, 878-882.

Poline, J.B., Holmes, A., Worsley, K., \& Friston, KJ. (1997). Making statistical inferences. In R,S.J. Frackowiak, KJ. Friston, CD. Frith, RJ Dolan, \& J.C Mazziotta (Eds.), Human brain function (pp. 85-106), San Diego: Academic Press.

Pollack, L, Johnson, L.B., \& Knaff, P.R. (1959). Running memory span. Journal of Experimental Psychology, 57, 137-146.

Salmon, E., Van der Linden, M., Collette, F., Delfiore, G., Maquet, P., Degueldre, C, Luxen, A., \& Franck,G. (1996). Regional brain activity during working memory tasks. Brain, 119, 1617-1625. Smith, E.E., \& Jonides, J. (1997). Working memory: A view from neuroimaging. Cognitive Psychology, 33, 5-42.

Smith, E.E., Jonides, J., \& Koeppe, R.A. (1996). Dissociating verbal and spatial working memory using PET. Cerebral Cortex, 6, 11-20.

Talairach, J., \& Tournoux, P. (1988). Co-planar stereotaxic atlas of the human brain: 3 -

dimensional proportional system: An approach to cerebral imaging. Stuttgart: Thieme. Vallar, G., \& Baddeley, AD. (1984). Fractionation of working memory: Neuropsychological

evidence for a phonological short-term store. Journal of Verbal Learning and Verbal Behavior, 23, 151-161.

Van der Linden, M., Brédart, S., \& Beerten, A. (1994), Age-related differences in updating working memory. British Journal of Psychology, 85, 145-152.

Woods, R.P., Cherry, S.R., \& Mazziotta, J.C. (1992). Rapid automated algorithm for aligning and reslicing PET images. Journal of Computed Assisted Tomography, 16, 620-633. 